\title{
Forsterite Formed on MgO Single Crystals During In-Situ Annealing
}

\author{
J. Bentley, ${ }^{*}$ J.K. Farrer, ${ }^{\ddagger \S}$ M.T. Johnson, ${ }^{\dagger \dagger}$ and C.B. Carter ${ }^{\ddagger}$ \\ *Metals \& Ceramics Div., Oak Ridge National Laboratory, PO Box 2008, Oak Ridge, TN 37831-6064 \\ ${ }^{\ddagger}$ Chemical Engineering and Materials Science, University of Minnesota, Minneapolis, MN 55455 \\ ${ }^{\S}$ Now at: Dept. of Physics \& Astronomy, Brigham Young University, Provo, UT 84602 \\ †Now at: Seagate Technology, 7801 Computer Avenue, Bloomington, MN 55435
}

Wang et al. previously annealed $\mathrm{MgO}$ in the TEM to $1500 \mathrm{~K}$, observed a phase transformation at the surface, and identified the phase as $\mathrm{MgO}_{2}$ [1]. They primarily studied bulk, cleaved $\{100\}$ surfaces in the reflection geometry (REM, RHEED, and REELS), but also studied thin cleavage fragments by transmission electron microscopy (TEM), selected area diffraction (SAD), and transmission electron energy-loss spectroscopy (EELS). In light of the extensive use of annealed single crystal $\mathrm{MgO}$ as a substrate for epitactic growth, numerous previous studies of $\mathrm{MgO}$ surfaces, and especially the seemingly unlikely formation of $\mathrm{MgO}_{2}$ (stable only at high oxygen partial pressures) in the vacuum of the TEM, additional in-situ annealing studies have been performed. Single-crystal $\{001\} \mathrm{MgO}$ TEM specimens, prepared following an involved and proven protocol [2], were annealed in situ with the use of a Gatan model 628 single-tilt heating holder. Most anneals employed a Philips CM30; additional anneals used an oil-free, dry-pumped Philips CM200-FEG. Specimens were heated in situ to $\sim 1500 \mathrm{~K}$ at $\sim 1 \times 10^{-7}$ Torr, held at that temperature for a few minutes, and cooled. Other than preliminary experiments to determine appropriate conditions, in-situ anneals were performed with specimens kept out of the electron beam. Comparison specimens were annealed in a vacuum furnace at $10^{-5}$ to $10^{-6}$ Torr, but no second phase formed.

In figure 1, the weak-beam image shows a contamination-free, step-terrace structure, the SAD pattern shows only expected $\mathrm{MgO}$ reflections, and the EELS shows only $\mathrm{O}$ and $\mathrm{Mg}$ edges, quantified with Gatan EL/P software to yield $\mathrm{Mg} / \mathrm{O}=1.0 \pm 0.1$. Following in-situ annealing (figure 2) the bright-field image shows $\sim 100$-nm grains on the specimen surfaces, the SAD pattern has the same features as in Wang et al. [1] (extra reflections at 3/2\{100\} (type 1) and $\{110\}$ (type2) plus double diffraction), and EELS shows $\mathrm{Si}$, possibly $\mathrm{Al}$, and lower $\mathrm{Mg}$ relative to $\mathrm{O}$. Quantification yields $\mathrm{Mg} / \mathrm{O}=0.50 \pm 0.05$ and $\mathrm{Si} / \mathrm{Mg}=0.49 \pm 0.05$, consistent with forsterite $\mathrm{Mg}_{2} \mathrm{SiO}_{4}$. Upon additional annealing in air at $1623 \mathrm{~K}$ for $15 \mathrm{~min}$ the polycrystalline film dewets the surface and forms small islands with moiré fringes visible on some particles [3]. Large-area SAD patterns exhibit the same features as after in-situ annealing, except 4-fold spots around $\mathrm{MgO}$ reflections (possibly $<111>$ relrods from defects on $\{111\}$ ) are no longer present. Tilting experiments (on carefully selected individual particles) around $1 \overline{3} 0_{\mathrm{f}}$ (type 1$) / / 200_{\mathrm{M}}$ reflections to [312] $\left(10^{\circ}\right)$ and [311] $\left(25^{\circ}\right)$ forsterite zones and around $002_{\mathrm{f}}$ (type 2$) / / 220_{\mathrm{M}}$ reflections to [210] $\left(5^{\circ}\right),[310]\left(16.5^{\circ}\right)$ and [410] $\left(24^{\circ}\right)$ forsterite zones, determined the orientation relationship as $(100)_{\mathrm{f}} / /(\overline{1} 11)_{\mathrm{M}},(010)_{\mathrm{f}} / /(\overline{2} 1 \overline{1})_{\mathrm{M}}$ and $(001)_{\mathrm{f}} / /(011)_{\mathrm{M}}(12$ variants $)$, where subscripts $\mathrm{f}$ and $\mathrm{M}$ refer to forsterite and $\mathrm{MgO}$, respectively [3]. Notable d-spacing matches are $\mathrm{d}_{400}$ forsterite $\sim \mathrm{d}_{222} \mathrm{MgO}$ and $\mathrm{d}_{004}$ forsterite $\sim \mathrm{d}_{220} \mathrm{MgO}$.

Possible sources of Si contamination were considered. The SHaRE CM30 always used silicon-free vacuum oils and greases. Furthermore, identical results were obtained with the SHaRE CM200-FEG specially equipped with oil-free "dry" vacuum pumps. The source of the Si was eventually traced to silicate sintering aids in the ceramic used to embed and electrically isolate the Pt heating element in the Gatan model 628 single-tilt heating holder used in the present [3] and earlier [1] work. The need to be aware of the details of the hardware used for in-situ experiments as a source of potential artifacts, and the usefulness of comparative ex-situ experiments cannot be underestimated [4].

1. Z.L. Wang, J. Bentley, E.A. Kenik, L.L. Horton and R.A. McKee, Surf. Sci. 273 (1992) 88-108.

2. M.G. Norton, S.R. Summerfelt and C.B. Carter, Appl. Phys. Lett. 56 (1990) 2246-8.

3. J.K. Farrer, M.T. Johnson, J. Bentley and C.B. Carter, Surf. Sci. 587 (2005) 205-18. 
4. Research at the ORNL SHaRE User Facility (JB) was supported by the Office of Basic Energy Sciences, US Department of Energy, under contract DE-AC05-00OR22725 with UT-Battelle, LLC. Research at the University of Minnesota (JKF, MTJ, CBC) was supported by US Department of Energy Grants DE-FG02-92ER45465-A004 and DE-FG02-01ER45883.
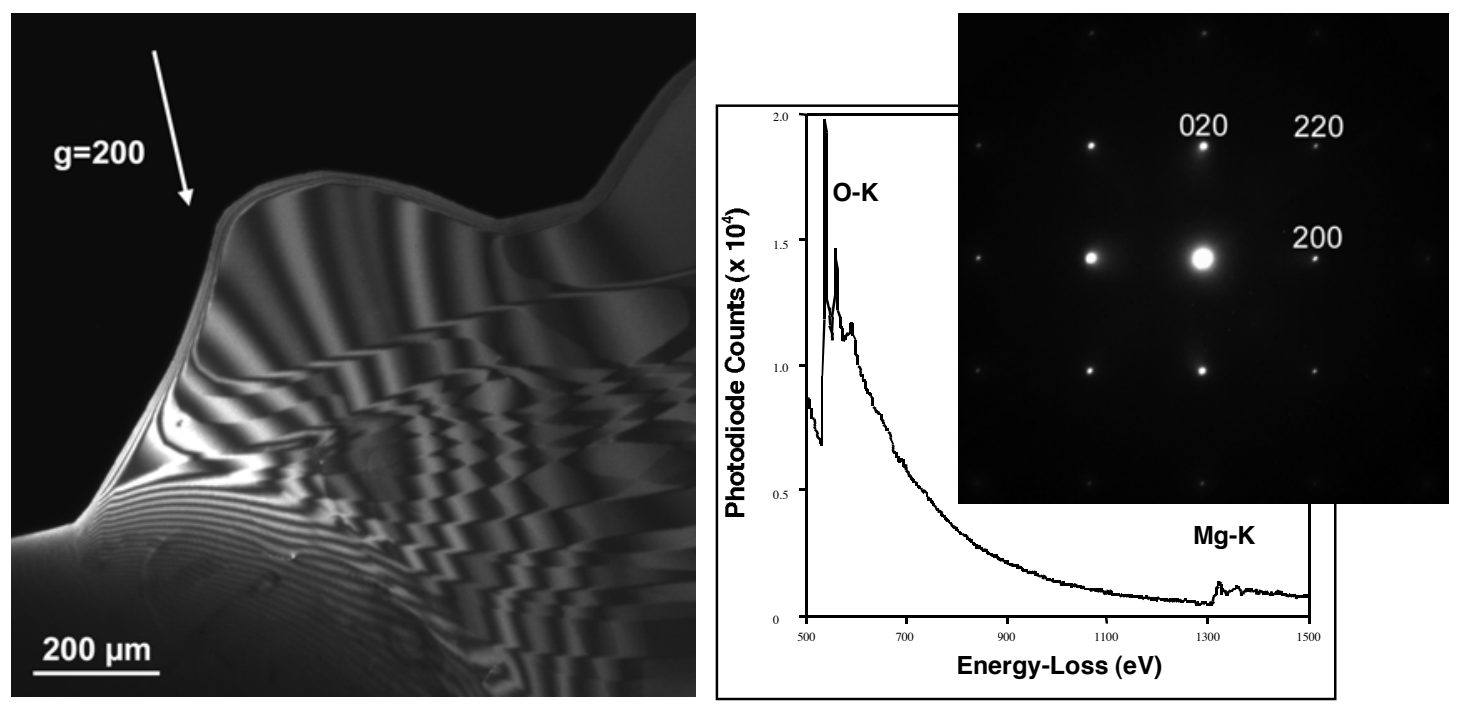

Fig. 1. Characterization of $\mathrm{MgO}$ specimen before in-situ annealing. Weak-beam dark-field image showing steps and terraces. [001] MgO SAD pattern showing only expected reflections. EELS data showing $\mathrm{O}$ and $\mathrm{Mg} \mathrm{K}$-edges quantified to yield $\mathrm{O} / \mathrm{Mg}=1.0 \pm 0.1$.
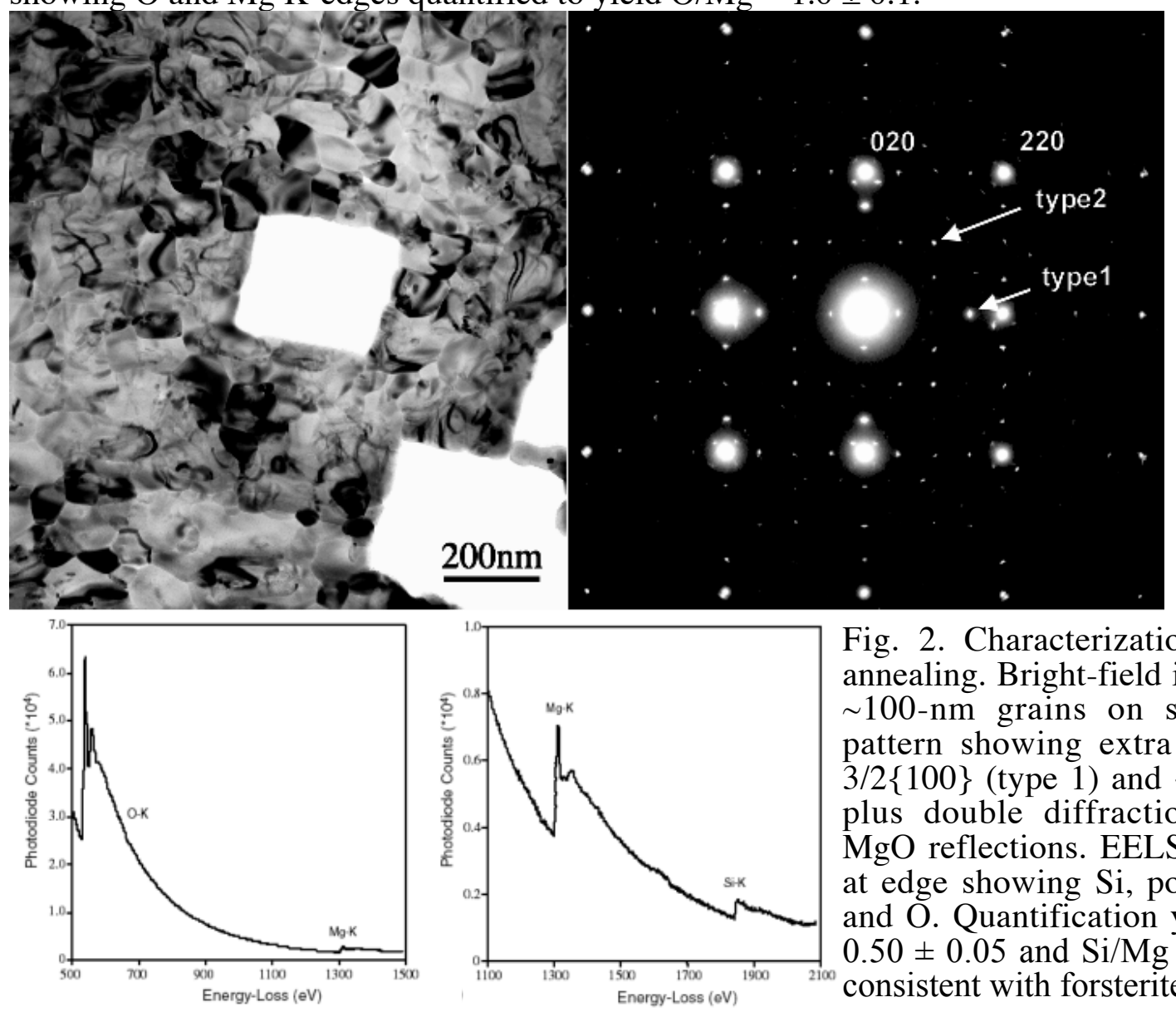

Fig. 2. Characterization after in-situ annealing. Bright-field image showing $\sim 100-$ nm grains on surfaces. SAD pattern showing extra reflections at $3 / 2\{100\}$ (type 1 ) and $\{110\}$ (type 2 ) plus double diffraction with [001] $\mathrm{MgO}$ reflections. EELS from particle at edge showing $\mathrm{Si}$, possibly $\mathrm{Al}, \mathrm{Mg}$ and $\mathrm{O}$. Quantification yields $\mathrm{Mg} / \mathrm{O}=$ $0.50 \pm 0.05$ and $\mathrm{Si} / \mathrm{Mg}=0.49 \pm 0.05$, consistent with forsterite $\mathrm{Mg}_{2} \mathrm{SiO}_{4}$. 